\title{
Contribution of MRI in Extension Assessment of Cervical Cancer in Yaoundé (Cameroon)
}

\author{
Mbozo'o Mvondo Samuel1,2*, Yannick Onana1, Aminou Mohamadou1, Jérémie Mbo Amvene1, \\ David Ngaroua1, Genesis Eric Secvou', Mathurin Neossi Guena', Obi Fidelis Ayuk ${ }^{3}$, Ekobena \\ Fouda $^{2}$, Odile Fernade Zeh4, Boniface Moifo ${ }^{4}$, Hamadou Ba1 ${ }^{1}$, Samuel Nko'o Amvene ${ }^{4}$
}

\footnotetext{
${ }^{1}$ Departement of Radiology and Medical Imaging, Faculty of Medicine and Biomedical Sciences of Garoua, University of Ngaoundéré, Ngaoundéré, Cameroon

${ }^{2}$ Department of Biomedical Sciences, Faculty of Sciences, University of Ngaoundéré, Ngaoundéré, Cameroon

${ }^{3}$ Department of Radiology, Yaoundé Military Hospital, Yaoundé, Cameroon

${ }^{4}$ Faculty of Medicine and Biomedical Sciences, University of Yaoundé, Cameroon

Email: ${ }^{\star}$ smbozoo@hotmail.com
}

How to cite this paper: Samuel, M.M., Onana, Y., Mohamadou, A., Amvene, J.M., Ngaroua, D., Secvou, G.E., Guena, M.N., Ayuk, O.F., Fouda, E., Zeh, O.F., Moifo, B., Ba, H. and Amvene, S.N. (2021) Contribution of MRI in Extension Assessment of Cervical Cancer in Yaoundé (Cameroon). Open Journal of Radiology, 11, 151-159. https://doi.org/10.4236/ojrad.2021.114014

Received: September 12, 2021

Accepted: November 22, 2021

Published: November 25, 2021

Copyright (c) 2021 by author(s) and Scientific Research Publishing Inc. This work is licensed under the Creative Commons Attribution International License (CC BY 4.0).

http://creativecommons.org/licenses/by/4.0/ (c) (i) Open Access

\begin{abstract}
Background: Cervical cancer is one of the most common cancers, requiring a precise complementary assessment. The aim of this study was to contribute to the improvement of the management of cervical cancer by describing the MRI aspects of cervical cancer according to the IFOG-MRI classification. Material and Methods: This was a cross-sectional study, conducted in patients who came for clinical suspicion, extension assessment of histologically confirmed cervical cancer, from October 2020 to March 2021, in Yaoundé (Cameroon). Results: 54 patients were selected, with an average age of 50.4 years. Genital hemorrhage was the most frequent symptom (92\%). The mean tumor size was $52.9 \mathrm{~mm}$. According to IFOG staging, stages Ia, Ib, IIa, IIb, IIIa, IIIb, and IV accounted for $9.10 \%, 10.20 \%, 12.50 \%, 29.50 \%, 5.70 \%, 12.50 \%$, and $20 \%$ respectively. No patient had known metastasis at the time of examination. Conclusion: MRI allows non-invasive, satisfactory tumor staging examination by providing information on tumor size, parametrium invasion, adjacent organ involvement, and the presence of lymph node metastasis.
\end{abstract}

\section{Keywords}

Cervical Cancer, Magnetic Resonance Imaging, International Federation of Obstetricians and Gynecologists

\section{Introduction}

Cervical cancer is one of the most common cancers, responsible for nearly 10 
million deaths in 2020 according to the data of World Health Organization [1]. It is also a public health problem in developing countries, especially in sub-Saharan Africa where it occupies the first rank among women's cancers [2]. In Cameroon, in 2018, there were an estimated 2350 cervical cancers and 1540 deaths per year, according to the data of GLOBOCAN [3].

The occurrence of cervical cancer is primarily related to infection with oncogenic Human Papillomavirus (HPV). Other predisposing factors include early sexuality, multiple sexual partners, early conception, smoking, and immunosuppression [4].

The staging of the disease has always been determined by the International Federation of Obstetricians and Gynecologists (IFOG) classification, which does not take into account some important parameters such as lymph node involvement [5].

Very often, the prognosis remains poor due to late diagnosis and treatment that is often inappropriate to the stage of the disease that could have been done by earlier MRI [2].

Though the MRI remains a very cost and difficult accessible exam in our country, does it efficiently participate in the management of cervical cancer?

With the general aim of participating in the improvement of cervical cancer management in Cameroon, this study was carried out to precise the role of pelvic Magnetic Resonance Imaging (MRI) for the characterization of the tumor, its local and lymph node extension and its complementarity to the IFOG classification in Cameroon [6].

\section{Material and Methods}

\subsection{Type of Study}

It was a cross-sectional, descriptive study, over a period of 6 months (from October 8, 2020 to March 21, 2021), carried out in the Radiology and Medical Imaging Department of the Military Hospital of Yaoundé (HMY).

\subsection{Study Population}

The population consisted of patients admitted to the MRI department of the Yaounde Military Hospital (YMH) for pelvic or abdominal-pelvic MRI for clinical suspicion, or extension assessment of histologically confirmed cervical cancer, or for follow-up of treated cervical cancer. Patients with known renal insufficiency or contraindication to contrast media injection were excluded from our study.

\subsection{Procedure}

The magnetic resonance imaging protocol included T2 Blade sequences in the three planes, T1 Dixon, T1 FS, diffusion, axial and coronal TSE T2 sequences on the abdomen (lymph node exploration), as well as T1FS sequences in the three planes after contrast injection. The slice thickness was $4-5 \mathrm{~mm}$, and the field of 
exploration covered from the recto-sigmoid hinge to the pubic symphysis, including the iliac lymph nodes. The injected contrast medium was $50 \mathrm{cc}$ of Gadolinium chelates.

We retrieved from the medical records on presenting complaints, initial clinical examination, MRI reports and images.

All MRI were reviewed by experienced radiologists.

\subsection{Variables}

The primary variables in this study were the pelvic MRI result. The imaging data collected were the presence of the tumor, its size, and the characterization of the tumor stage according to the IFOG classification [5] as well as lymph node involvement.

A tumor localized to the cervix was considered as stage I, a tumor with extension to parametrium and/or to the upper $1 / 3$ of the vagina was stage II tumor, a tumor reaching the pelvic wall and/or lower $1 / 3$ of the vagina and/or with presence of hydronephrosis was a stage III tumor, and the tumor with extension beyond the pelvis or attenuating the bladder and/or rectum was a stage IV tumor [5].

Secondary variables were socio-demographic data (age, geographical distribution, marital status, occupation), and clinical signs and symptoms.

\subsection{Statistical Analysis}

The data collected was processed through the following software: Microsoft Excel 2016 and IBM SPSS 23. The results are expressed in tables and figures, as mean and percentage.

\section{Results}

\subsection{Study Population}

A total of 54 patients were included in our study, with a mean age of 50.5 years. The most represented age group was 50 - 64 years $(38.90 \%, \mathrm{n}=21)$, as shown in Figure 1. 55.60\% $(n=30)$ of these patients lived in rural area, $59.6 \%(n=31)$ of them were married, and $25 \%(n=15)$ were widows. Housewives represented $51.9 \%$ of the patients $(n=28)$.

\subsection{Clinical Data}

The vast majority of our patients $(55 \%, \mathrm{n}=30)$ consulted on average 6 months after the onset of symptoms.

Table 1 shows the different functional signs, which were dominated by metrorragia $(92.5 \%, \mathrm{n}=50)$.

\subsection{Pelvic MRI Findings}

Figure 2 summarizes the IFOG tumor stages obtained in the 54 patients of our study population. Stage IIB was the most encountered $(29.5 \%, \mathrm{n}=26)$. 
Table 1. Distribution of cases by clinical signs.

\begin{tabular}{ccc}
\hline Clinical manifestations & Number & Percentage \\
\hline Metrorragia & 50 & $92.5 \%$ \\
Pelvic pain & 15 & $26.7 \%$ \\
Leucorrhoea & 08 & $15.7 \%$ \\
Other signs & 06 & $11.1 \%$ \\
\hline
\end{tabular}

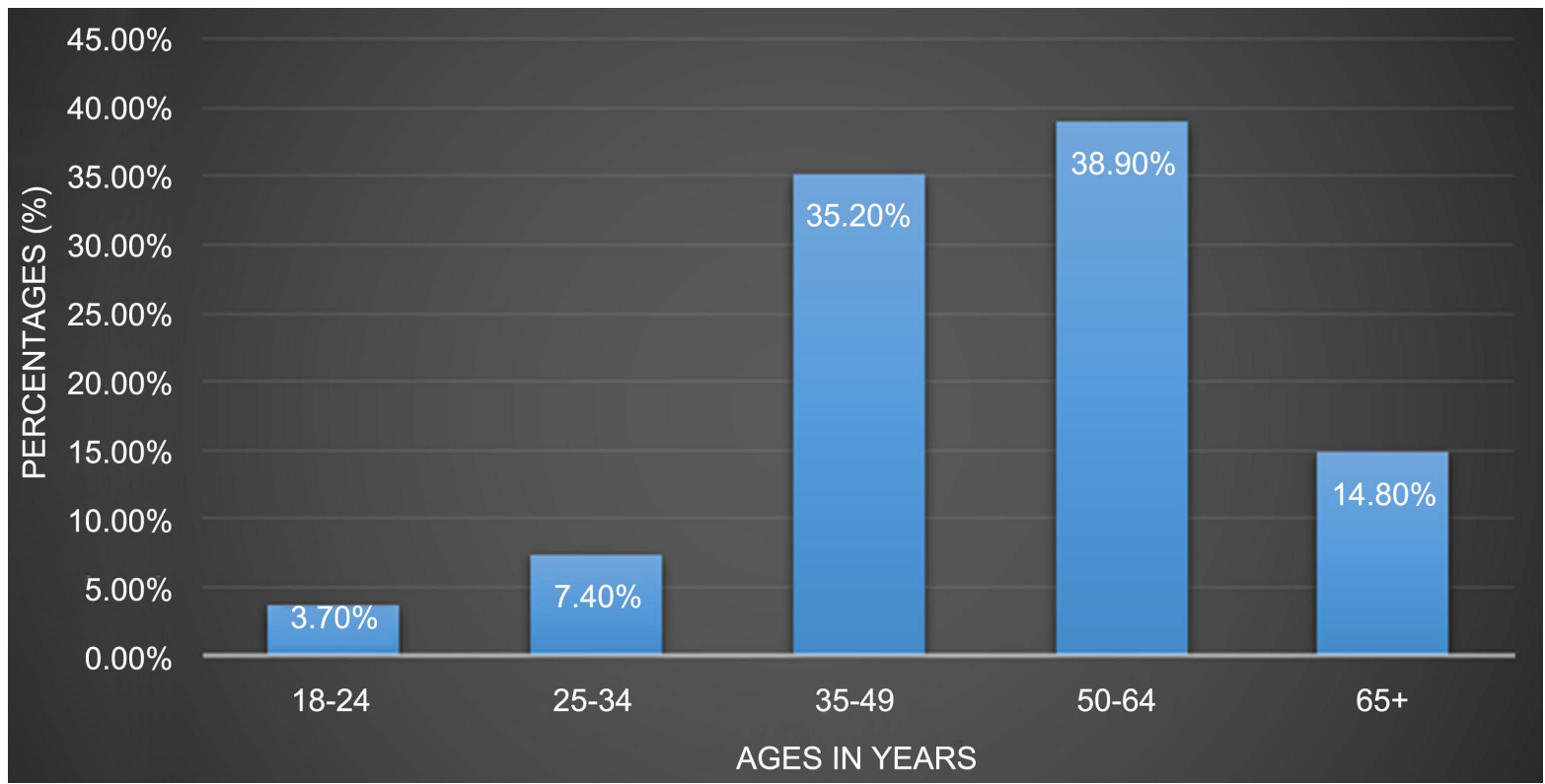

Figure 1. Age distribution.

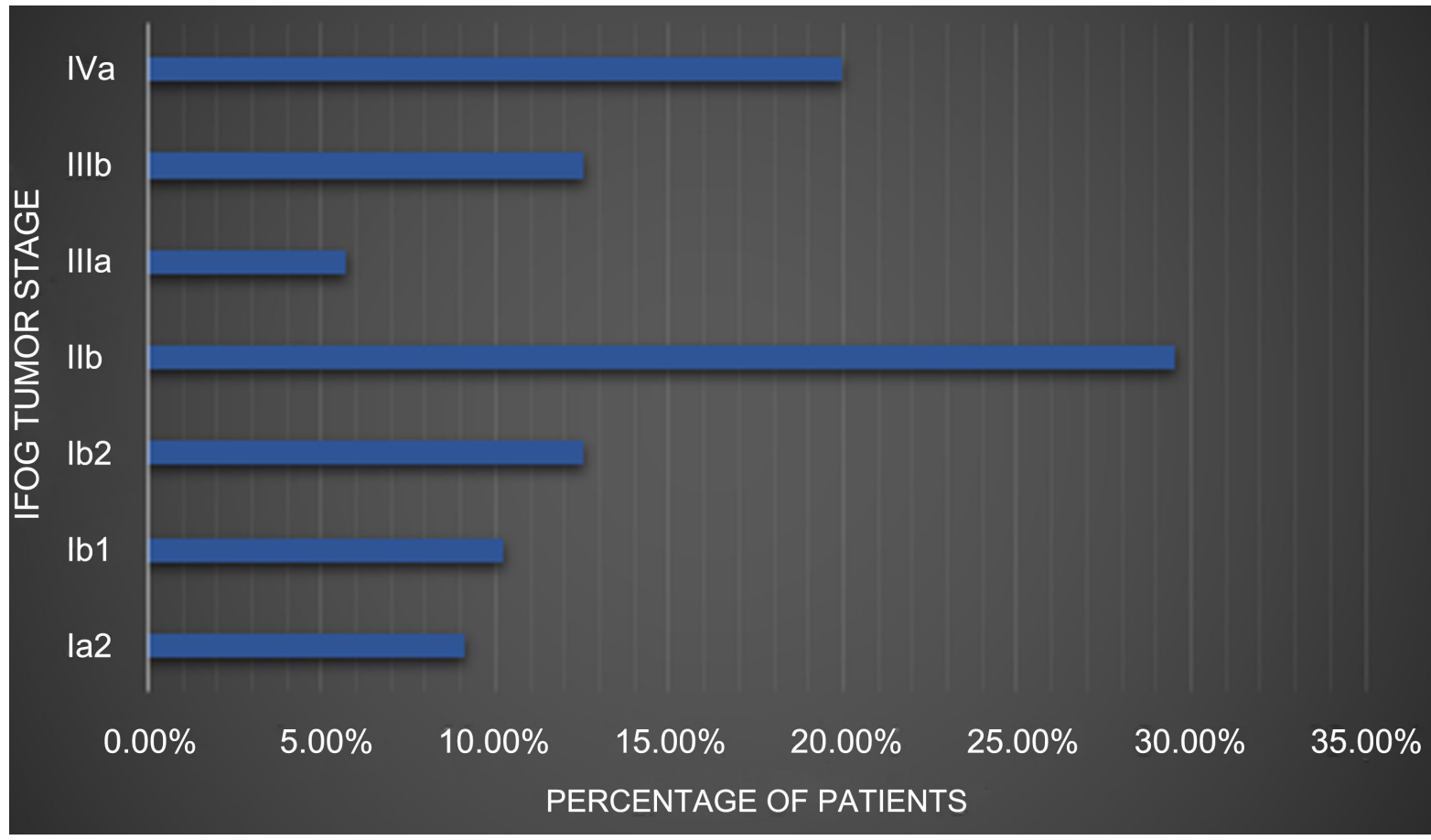

Figure 2. Distribution of patients according to IFOG tumors stages. 
Tumors limited to the cervix accounted for $26.7 \%(n=14)$. Parametrium invasion was unilateral in $13.6 \%(n=12)$ patients. Tumors involving the pelvic wall with associated hydronephrosis were present in $12.5 \%$ of cases $(n=11)$.

Lesions involving the bladder and/or rectum were present in $20.4 \%$ of cases (n $=18$ ), as shown in Figure 3. No patient had metastasis at the time of examination.

Concerning lymph node involvement, the internal and external iliac chains were invaded in $33.3 \%$ ( $n=18$ patients), followed by the retroperitoneal areas in $20.4 \%(\mathrm{n}=11)$.

Figure 4 and Figure 5 present some illustrative stage of cervical cancer.

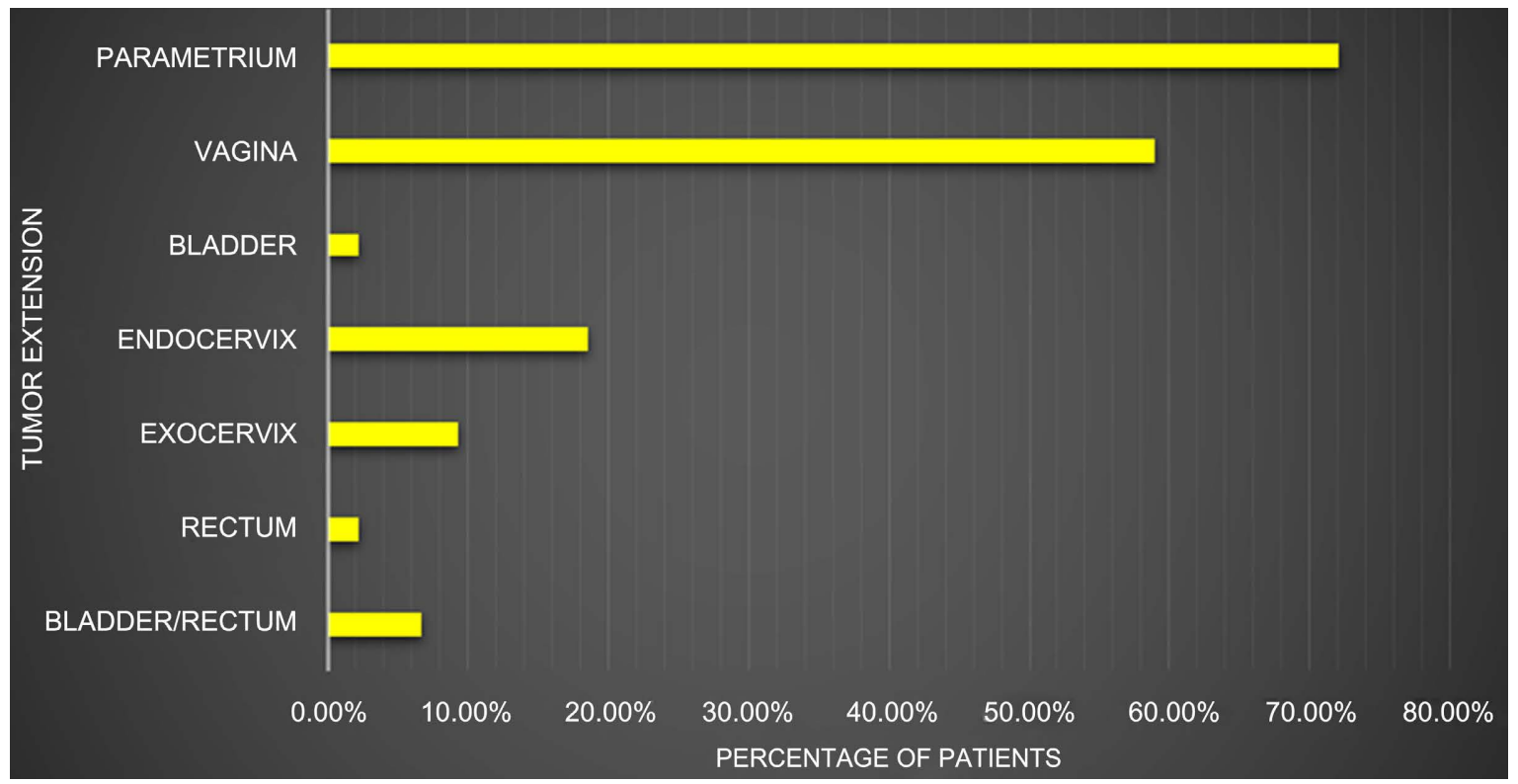

Figure 3. Distribution of patients according to loco-regional extension of tumors.

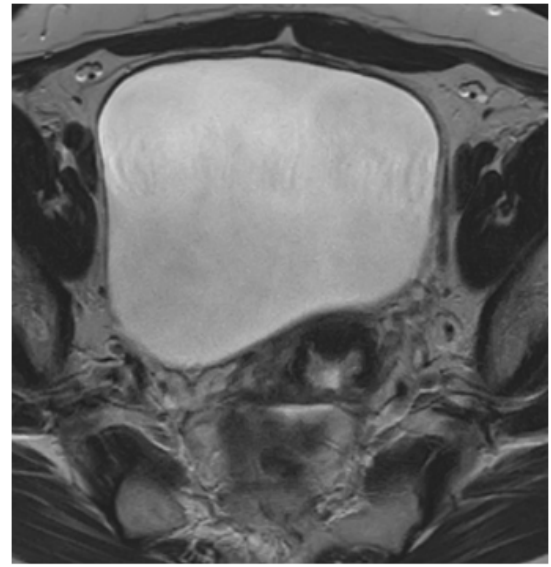

(A)

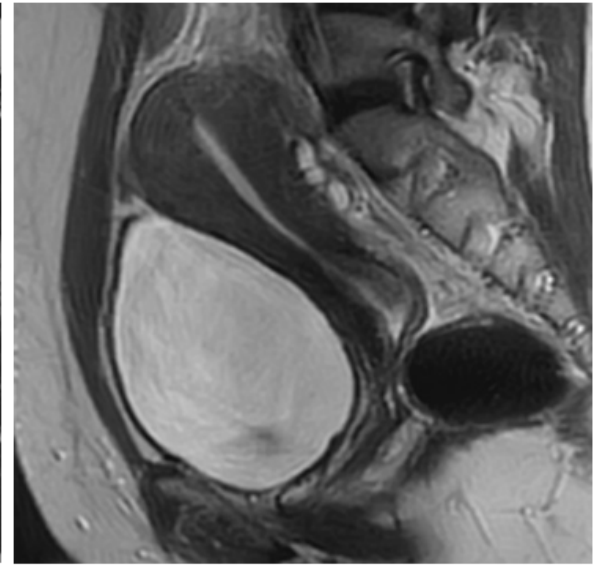

(B)

Figure 4. Cervical cancer classified as stage IB, tumor lesion of the posterior lip of the cervix. This Pelvic MRI of a 39-year-old woman, T2-weighted axial (A) and T2 sagittal (B) sequences, showing poorly delineated tissue area invading the stroma of the posterior lip of the cervix in intermediate $\mathrm{T} 2$ signal. 


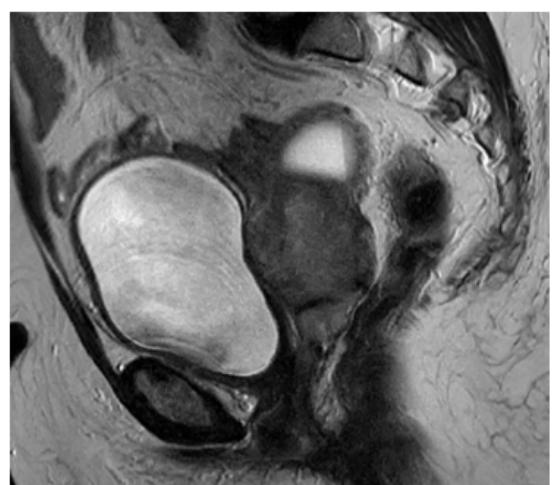

(A)

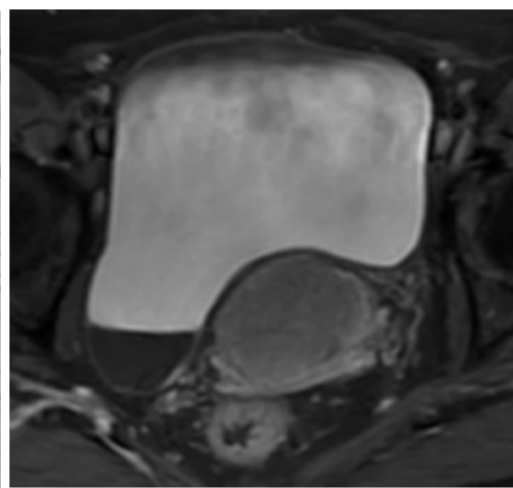

(B)

Figure 5. Cervical cancer classified as stage IIB IFOG classification Pelvic MRI of a 61-year-old woman, sagittal T2-weighted (A) and axial T1 FS + Gadolinium (B) sequences: Cervical lesion $(46 \times 41 \mathrm{~mm} \times 40 \mathrm{~mm}$ height $)$, poorly delimited, intermediate $\mathrm{T} 2$ signal, heterogeneously enhanced after IV PDC. It crosses the isthmus and invades the upper part of the vagina. Hydrometry is retained, and bilateral parametrial invasion, predominantly on the left.

\section{Discussion}

Cervical cancer is the most common cancer in Cameroon, as well as the leading cause of cancer death in women, as in most sub-Saharan African countries, which do not have a nationwide mass screening program [2] [3].

\subsection{Study Population}

In our series, the mean age at diagnosis was 50.5 years. The age group most affected was $50-64$ years (38.90\%). This result is similar to that of Aboubacar Keita et al. who found an age of 52 years in Mali [7], and differs moderately from that of El Ghorfi in Morocco et al. who found an age of 59 years [6]. However, in general, the early onset of cancer in sub-Saharan Africa has already been highlighted by many authors [2] [8].

Most of our patients lived in rural areas (55.60\%), as pointed out by Faye et al., who found a greater proportion of women living in rural areas (84.4\%) in Senegal [9], the rural area is characterized by the scarcity of screening and awareness campaigns.

\subsection{Clinical Data}

The major revealing sign was essentially metrorragia found in $92 \%$ of cases, which could be provoked or spontaneous. This result is similar to what Aboubacar et al. found in Mali metrorragia in $98.8 \%$ of cases [7].

According to our results, the delay of consultation is long, 55\% of the patients consulted after 6 months of evolution, similar to the results of Kouskous et al. who found $57.9 \%$ in the city of Fez [10].

\subsection{Pelvic MRI Findings}

The MRI maximum tumor diameter in this study was $52.9 \mathrm{~mm}(30-79 \mathrm{~mm})$ on 
average. This differs slightly from the results of El Ghorfi et al., who founded 61 $\mathrm{mm}[6]$.

Although the estimation of tumor size remains clinical, MRI is of major contribution to improve the definition with an accuracy of $95 \%$ [4].

Paramedic invasion represented $72.2 \%$ of cases. Our data are similar to those of Kouskous et al. in Fez who found 68\% parametrial invasion [10].

As pointed out by Shweel et al., parametrial invasion is an important criterion in the preoperative workup [11] [12]. However, there is a risk of overestimation due to peritumoral edema in the case of voluminous involvement [11] [13] [14].

MRI has a specificity of $97 \%$, and a negative predictive value of $100 \%$ for parametrial invasion [15].

Regarding the classification used, which was that of IFOG, we obtained the following results: stage IA $(9.10 \%)$, stage IB (10.20\%), stage IIA (12.50\%), stage IIB (29.50\%), stage IIIA (5.70\%), stage IIIB (12.50\%), and stage IVA (20\%).

Similar proportions in severe forms were found by Ghorfi et al. (32\%) [6] and Shweel et al. in Egypt (26.6\%) [11].

This similarity of severe forms could be explained by the difficulties encountered in the health systems of developing countries in terms of mass screening, particularly in Cameroon where $62.9 \%$ of cervical cancers are diagnosed at an advanced stage [16].

Concerning lymph node involvement, retroperitoneal areas were invaded in $20.4 \%$ of cases, which is a poor prognostic factor, and is equivalent to extrapelvic tumor extension (stage IVB), according to some authors [11] [17].

The limitation of this study was the small size of our sample, probably due to the monocentric nature of our work.

\section{Conclusions}

MRI has an important contribution to the evaluation of cervical cancer. It has been shown to complement the clinical examination by providing information on tumor size, parametrial invasion, adjacent organs involvement, and the presence of lymph node metastasis. Thus, MRI allows non-invasive is satisfactory in tumor staging.

Therefore, IRM is the reference examination for planning, choice of treatment, and search for possible tumor recurrence [7]. However, its relatively high cost and poor accessibility in our geographical context seem to be limiting factors, as in Cameroon.

A larger size of sample study would help to generalize our results in our context.

\section{Author Contributions}

All authors contributed to the writing, read and approved the final version of the manuscript.

\section{Acknowledgements}

We thank all the radiologists and technicians of the radiology department of the 
Military Hospital of Yaoundé who contributed to the collection of data analyzed in this study.

\section{Conflicts of Interest}

The authors declare no conflict of interest.

\section{References}

[1] Cancer. https://www.who.int/fr/news-room/fact-sheets/detail/cancer

[2] Muteganya, D., Bigayi, T., Bigirimana, V., Sindayirwanya, J.B. and Marerwa, G. (1999) Cervical Cancer at Kamenge University Hospital about 35 Cases. Médecine d Afrique Noire, 46, 153-156. http://www.santetropicale.com/Resume/34606.pdf

[3] Tebeu, P.M., Antaon, J.S.S., Adjeba, M., Pikop, F., Fouogue, J.T. and Ndom, P. (2020) Knowledge, Attitudes and Practices of Health Professionals on Cervical Cancer in Cameroon. Public Health, 32, 489-496. https://doi.org/10.3917/spub.205.0489

[4] Thille, A. (2004) Cervical Cancers: What Can MRI Do? Journal of Radiology, 85, 1366. https://doi.org/10.1016/S0221-0363(04)77185-1

[5] IGOF Classification of Cervical Carcinomas. https://screening.iarc.fr

[6] El Ghorfi, S. (2020) Contribution of MRI in the Assessment of Loco-Regional Extension of Cervical Cancer in 28 Cases. http://ao.um5.ac.ma/jspui/bitstream/123456789/18711/1/MS1762020.pdf

[7] Balleyguier, C., Kinkel, K., Haie-Meder, C., Morice, P., Lhomme, C., Lumbroso, J., et al. (2004) Which Examinations Should Be Proposed for the Assessment of Extension and Follow-Up of Uterine Cervical Cancers: MRI, CT, PET-CT. Journal of Radiology, 85, 1190. https://doi.org/10.1016/S0221-0363(04)76605-6

[8] Keita, A. (2020) Cervical Cancer: Therapeutic Aspect in the Radiotherapy Department of the Hôpital du Mali. https://www.bibliosante.ml/bitstream/handle/123456789/3797/20M24.pdf

[9] Alliance of Francophone African and Mediterranean Leagues against Cancer (2017) Cancer in Francophone Africa. https://www.iccp-portal.org/system/files/resources/LivreCancer.pdf

[10] Faye, A., Mbengue, E.T. and Tal-Tal, A. (2017) Factors Associated with Cervical Cancer Screening in Rural Senegal. Journal of Epidemiology and Public Health, 65, S77. https://doi.org/10.1016/j.respe.2017.03.063

[11] Kouskous, F. (2012) Cervical Cancer: Anatomopathological Studies (About 86 Cases).

http://www.chu-fes.ma/cancer-du-col-uterin-etudes-anatomopathologiques-a-prop os-de-86-cas

[12] Shweel, M.A., Abdel-Gawad, E.A., Abdelghany, H.S., Abdel-Rahman, A.M. and Ibrahim, E.M. (2012) Uterine Cervical Malignancy: Diagnostic Accuracy of MRI with Histopathologic Correlation. Journal of Clinical Imaging Science, 2, 42. https://doi.org/10.4103/2156-7514.99175

[13] Jena, A., Oberoi, R., Rawal, S., Das, S.K. and Pandey, K.K. (2005) Parametrial Invasion in Carcinoma of Cervix: Role of MRI Measured Tumour Volume. British Journal of Radiology, 78, 1075-1077. https://doi.org/10.1259/bjr/36116150

[14] Nicolet, V., Carignan, L., Bourdon, F. and Prosmanne, O. (2000) MR Imaging of Cervical Carcinoma: A Practical Staging Approach. Radiographics, 20, 1539-1549. https://doi.org/10.1148/radiographics.20.6.g00nv111539 
[15] Sala, E., Wakely, S., Senior, E. and Lomas, D. (2007) MRI of Malignant Neoplasms of the Uterine Corpus and Cervix. American Journal of Roentgenology, 188, 1577-1587. https://doi.org/10.2214/AJR.06.1196

[16] Sahdev, A., Sohaib, S.A., Wenaden, A.E.T., Shepherd, J.H. and Reznek, R.H. (2007) The Performance of Magnetic Resonance Imaging in Early Cervical Carcinoma: A Long-Term Experience. International Journal of Gynecological Cancer, 17, 629-636. https://doi.org/10.1111/j.1525-1438.2007.00829.x

[17] Batoum, V.M., Sando, Z. and Nkegoum, B. (2020) Opportunistic Screening for Pre-Invasive Cervical Lesions and Cervical Cancer in Cameroonian Pregnant Women. Health Sciences and Disease. https://www.hsd-fmsb.org/index.php/hsd/article/view/1973 